\title{
MOBILIZACC̃̃O PRECOCE DE DOENTES NA UNIDADE CUIDADOS INTENSIVOS: CONTRIBUTO PARA A ENFERMAGEM DE REABILITAÇÃO. UMA REVISÃO SISTEMÁTICA DA LITERATURA
}

\author{
EARLY MOBILIZATION OF PATIENTS IN THE INTENSIVE CARE UNIT: CONTRIBUTION TO \\ REHABILITATION NURSING. A SYSTEMATIC REVIEW OF THE LITERATURE
}

\section{MOVILIZACIÓN TEMPRANA DE PACIENTES EN LA UNIDAD DE CUIDADOS INTENSIVOS: CONTRIBUCIÓN A LA ENFERMERÍA DE REHABILITACIÓN. UNA REVISIÓN SISTEMÁTICA DE LA LITERATURA}

\author{
Sandra Reis ${ }^{(1)}$, Eva Rosa Mendes Castro ${ }^{(2)}$, Sandrina Isabel Pimenta Carvalho ${ }^{(3)}$, Sónia Zulmira Ferreira Carvalho ${ }^{(4)}$, Carla Sílvia \\ Fernandes $^{(5)}$, Maria Manuela Ferreira Pereira Silva Martins ${ }^{(6)}$,
}

\footnotetext{
(1) Hospital da Senhora da Oliveira Guimarães, Guimarães, Portugal; (2) Hospital da Senhora da Oliveira Guimarães, Guimarães, Portugal; (3) Hospital Pedro Hispano, Matosinhos, Portugal; (4) Hospital da Senhora da Oliveira Guimarães, Guimarães, Portugal; (5) Escola Superior de Enfermagem do Porto, Porto, Portugal; (6) Escola Superior de Enfermagem do Porto (ESEP), Centro de Investigação em Tecnologias e Serviços de Saúde (CINTESIS), Porto, Portugal;
}

\section{Descritores}

Deambulação Precoce Mobilização Precoce Mobilidade precoce

Reabilitação

Enfermeiros

Enfermagem

Cuidados Intensivos

\section{Resumo}

Introdução: A mobilização precoce é uma técnica, que pode facilitar a realização dos autocuidados, promovendo ganhos em força muscular e um aumento do grau de independência, reduzindo consequentemente, o tempo de internamento assim como, os custos inerentes a ele.

Objetivos: Identificar através de um estudo de revisão sistemática da literatura os benefícios da mobilização precoce em doentes de cuidados intensivos.

Metodologia: Realizou-se uma revisão sistemática da literatura baseada na metodologia proposta pelo Joanna Briggs Institute. A pesquisa foi realizada nas bases de dados MedLine ${ }^{\circ}$, CINAHL $®$, PubMed $\circledast$ considerando como critérios de inclusão estudos publicados nos últimos 10 anos. Foram utilizados os instrumentos da JBI para avaliação metodológica dos estudos.

Resultados: Foram incluídos 5 estudos, de um total de 160 artigos identificados $A$ inatividade prolongada leva a inúmeras complicações, nomeadamente cognitivas, funcionais e emocionais, que através da mobilização precoce podem ser minimizadas e/ou evitadas.

Conclusão: Através da análise dos artigos verificamos que a mobilização precoce reduz o tempo de internamento e evita complicações e permite ganhos em saúde. Contudo, foram identificadas algumas barreiras tais como a carga de trabalho dos enfermeiros, a pouca clareza quanto à responsabilidade da mobilização, os riscos de lesões da equipa, a motivação do doente e a participação da família que fragilizam a mobilização precoce.

\section{Abstract}

Introduction: Early mobilization is a technique that can facilitate the performance of self-care, promoting gains in muscle strength and an increase in the degree of independence, consequently reducing the length of hospital stay as well as the costs inherent to it.

Objectives: To identify, through a systematic literature review study, the benefits of early mobilization in intensive care patients.

Methodology: A systematic literature review was carried out based on the methodology proposed by the Joanna Briggs Institute. The research was carried out in the MedLine $\AA$, CINAHL $₫$, PubMed $₫$ databases considering as inclusion criteria studies published in the last 10 years. JBI instruments were used to methodologically evaluate the studies.

Results: Five studies were included, out of a total of 160 identified articles. Prolonged inactivity leads to numerous complications, namely cognitive, functional and emotional, which through early mobilization can be minimized and / or avoided. 
Conclusion: Through the analysis of the articles, we found that early mobilization reduces the length of hospital stay and avoids complications and allows health gains. Yet, some barriers were identified, such as the workload of nurses, the lack of clarity regarding the responsibility for mobilization, the risks of injury to the team, the patient's motivation and the participation of the family that weakens early mobilization

\section{Descriptores}

Enfermería

Enfermeras

Movilización Precoz

Ambulación precoz

Rehabilitacion

Cuidados intensivos

\section{Resumen}

Introducción: La movilización precoz es una técnica que puede facilitar la realización del autocuidado, favoreciendo la ganancia de fuerza muscular y un aumento del grado de independencia, reduciendo consecuentemente la duración de la estancia hospitalaria así como los costes inherentes a la misma.

Objetivos: Identificar mediante un estudio de revisión de la literatura sistemática los beneficios de la movilización temprana en pacientes de cuidados intensivos.

Metodología: Se realizó una revisión de la literatura sistemática basada en la metodología propuesta por el Instituto Joanna Briggs. La investigación se realizó en las bases de datos MedLine $\circledast$, CINAHL $\circledast$, PubMed $\circledast$ considerando como criterios de inclusión los estudios publicados en los últimos 10 años. Se utilizaron instrumentos del JBI para evaluar metodológicamente los estudios.

Resultados: Se incluyeron cinco estudios, de un total de 160 artículos identificados. La inactividad prolongada conduce a numerosas complicaciones, cognitivas, funcionales y emocionales, que mediante una movilización precoz pueden minimizarse y / o evitarse.

Conclusión: A través del análisis de los artículos, encontramos que la movilización temprana reduce la duración de la estancia hospitalaria y evita complicaciones y permite mejoras en la salud. Todavía, se identificaron algunas barreras, como la carga de trabajo de las enfermeras, la falta de claridad en cuanto a la responsabilidad de la movilización, los riesgos de lesión al equipo, la motivación del paciente y la participación de la familia que debilita la movilización temprana.

\section{INTRODUÇÃO}

O ser humano é único e o seu movimento também o é, embora tenha caraterísticas fisiológicas semelhantes e compatíveis com alguma normalizacão desse movimento. Embora tendencialmente quando olhamos o movimento corporal da pessoa enquadramo-la basicamente como um aspeto fisiológico, ele tem interação psicológica e cultura apresenta-se de formas diferentes ao longo do ciclo de vida, tornando um aspeto da vida que concorre significativamente para a sobrevivência, sendo considerado uma função básica.

O ser humano usa o movimento de forma interativa consigo mesmo pelo que ele próprio é uma expressão de comunicação, mas também como suporte aos seus autocuidados e algumas funções vitais.

A capacidade de andar é uma atividade física fundamental para a realizacão de autocuidados e das atividades da vida diária, tornando-se um indicador relevante para a gestão da alta dos doentes internados existindo uma maior a probabilidade de alta precoce. São referidas inúmeras complicacõos decorrentes da não mobilização precoce designadamente, delírio, trombose venosa profunda, pneumonia, infecão do trato urinário, obstipacão, entre outras complicacões (Wang, Xiao, Zhang, Jia, \& Shi, 2020). A mobilização torna-se uma intervenção de enfermagem quando as pessoas estão sujeitas a imobilidade, detém incapacidade de várias origens para realizar os movimentos de forma independente, ocorrendo esta situação por várias patologias, particularmente no envelhecimento, mas também por situações induzidas como ocorre nos cuidados intensivos (Ordem dos Enfermeiros, 2013). Os enfermeiros de reabilitação pela formação especializada que tem tornam-se profissionais com competências para mobilizar com seguranca os doentes, mesmo que estes não tenham forca ou vontade de o fazer (DR, 2019). O enfermeiro especialista de reabilitação deve salientar os elementos determinantes da qualidade dos seus cuidados, numa perspetiva de melhoria contínua comunitários (Gomes, Martins, Gonçalves, \& Fernandes, 2012). A reabilitação introduzida precocemente melhora os resultados e ganhos em saúde da pessoa (Karic, Røe, Nordenmark, Becker, \& Sorteberg, 2016).

Sem o contributo da investigação, nenhuma profissão poderá conhecer um desenvolvimento contínuo, em que cada uma delas deve ser capaz de fornecer aos seus elementos profissionais uma base de conhecimentos teóricos sobre a qual assenta a sua prática, fornecer serviços de qualidade às pessoas e aos grupos (Gomes, Martins, Gonçalves, \& Fernandes, 2012). Pelo que, a presente revisão sistemática da literatura tem como objetivo a análise de estudos que respondam à questão de partida "Qual o contributo das mobilizacões precoces nos doentes em UCI, realizadas por enfermeiros de reabilitacão?". Com a elaboracão deste trabalho pretendemos identificar evidência científica para a tomada de decisão clínica e refletir na nossa prática de forma a integrar os resultados que emergirem deste estudo.

\section{METODOLOGIA}

Numa primeira fase pretendíamos explorar a questão do primeiro levante, contudo na pesquisa efetuada nas diferentes bases de dados, ao trabalharmos esse descritor não obtivemos quaisquer resultados. Como tal, alargamos a área de pesquisa tendo sobressaído o tema da mobilidade precoce como mais próximo do nosso objetivo. A escolha deste tema surgiu, pois, todos os autores envolvidos na análise deste percurso deparam-se diariamente, ao longo da sua prática clínica, com a relutância por parte dos elementos da equipa de enfermagem em se realizar uma mobilização precoce dos doentes, devido aos receios, infundados que poderemos considerar por fundamento o desconhecido. 0 nosso trabalho de pesquisa iniciou-se pela pergunta: Qual o contributo das mobilizações precoces nos doentes em UCl, realizadas por enfermeiros de reabilitação? Esperamos que no fim deste estudo passamos contribuir com conhecimento para a prática clínica. 
Foi realizada uma revisão sistemática da literatura durante o mês de novembro de 2020, através das bases: EBSCO (MedLine ${ }^{\circ}$, CINAHL $®$, PubMed $\circledast$ ) resultando uma recolha de 160 artigos. O processo de seleção dos artigos passou por uma leitura, analisados de forma independente por cada um dos investigadores obtendo-se consenso de 5 artigos que foram incluídos para análise. Dos critérios de inclusão fizeram parte: estudos publicados nos últimos 10 anos, artigos completos, artigos em inglês, português e italiano, sem exclusão de estudos.

Foi desenvolvida uma questão clínica segundo a metodologia PICO: População- Doentes de Unidade Cuidados Intensivos, Intervenção- Intervenção do enfermeiro de reabilitação na mobilização precoce, Comparação- Sem intervenção, OutcomesImpacto da mobilização precoce

Os descritores de pesquisa foram utilizados os descritores MESH e livres apresentados na tabela seguinte, o que nos permitiu a elaboração da frase Boleana: Descritores de Pesquisa (tabela 1).

Tabela 1 - Descritores de Pesquisa

\begin{tabular}{|l|l|}
\hline MESH & Livres \\
\hline Early Ambulation & Early Mobility \\
\hline Rehabilitation & Early Mobilization \\
\hline Nursing & Intensive Care \\
\hline Nurse & \\
\hline Nurses & \\
\hline
\end{tabular}

O processo de identificação e inclusão dos artigos foi realizado e encontra-se apresentado através do PRISMA (Preferred Reporting Items for Systematic Reviews and Meta-Analyses) (Figura 1). A avaliação metodológica dos estudos foi realizada através dos instrumentos propostos pela JBI. Dos 39 artigos selecionados para leitura integral, 5 estudos foram incluídos para amostra final. Os estudos foram codificados para facilitar a apresentação de resultados.
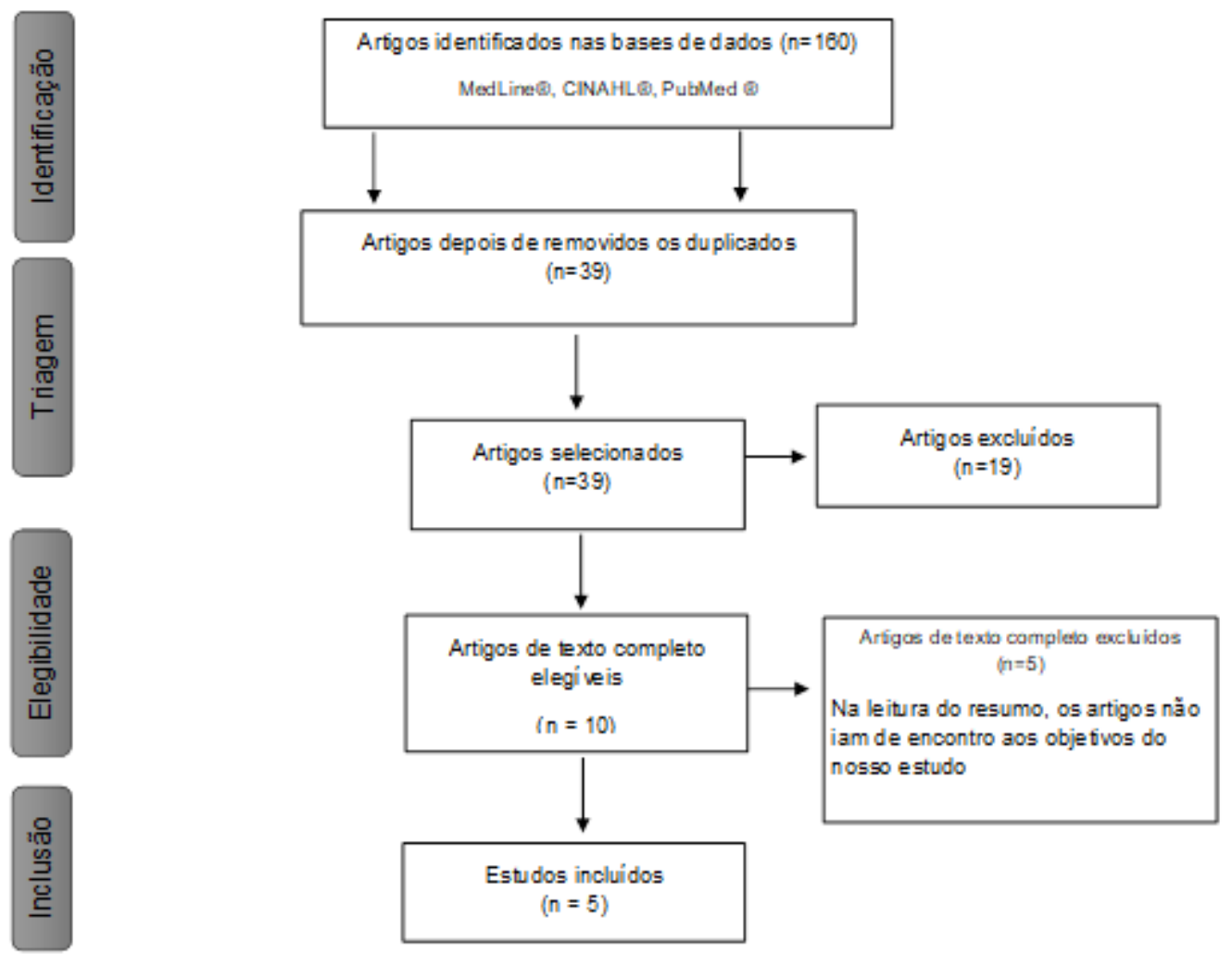

Figura 1 - Processo de identificação e inclusão dos artigos - PRISMA Diagram flow

\section{RESULTADOS}

A Tabela 2 resume as características dos 5 estudos integrados na revisão, no que se refere aos autores, ano, objetivos do estudo, desenhos de estudo e participantes. 
Tabela 2 - Caracterização dos estudos incluídos na revisão

\begin{tabular}{|c|c|c|c|c|c|}
\hline $\begin{array}{l}\text { Código } \\
\text { do } \\
\text { estudo }\end{array}$ & $\begin{array}{l}\text { Autores } \\
\text { Ano }\end{array}$ & País & $\begin{array}{l}\text { Desenho } \\
\text { Estudo }\end{array}$ & Objetivos/ Participantes & $\begin{array}{l}\text { Resultado do Score } \\
\text { JBI }\end{array}$ \\
\hline$\overline{E 1}$ & $\begin{array}{l}\text { Tempera, } \\
\text { Alfio et al. } \\
2013\end{array}$ & Itália & $\begin{array}{l}\text { Estudo de } \\
\text { Revisão } \\
\text { Sistemática }\end{array}$ & $\begin{array}{l}\text { Relação entre a mobilização precoce e a } \\
\text { prevenção da síndrome de desuso em } \\
\text { utentes com mais de } 65 \text { anos. } N=\text { não } \\
\text { especificado nos } 25 \text { estudos analisados }\end{array}$ & $\begin{array}{l}\text { Dos } 11 \text { itens totais, } 10 \\
\text { têm resposta positiva }\end{array}$ \\
\hline E2 & $\begin{array}{l}\text { Azevedo, } \\
\text { Gomes, } \\
2015\end{array}$ & Portugal & $\begin{array}{l}\text { Estudo de } \\
\text { Revisão } \\
\text { Sistemática }\end{array}$ & $\begin{array}{l}\text { Determinar os efeitos da mobilização } \\
\text { precoce na reabilitação funcional de doentes } \\
\text { internados em cuidados intensivos. } \mathrm{N}=541\end{array}$ & $\begin{array}{l}\text { Dos } 11 \text { itens totais, } 10 \\
\text { têm resposta positiva }\end{array}$ \\
\hline E3 & $\begin{array}{l}\text { Azevedo, et } \\
\text { al. } 2019\end{array}$ & Portugal & $\begin{array}{l}\text { Descritivo } \\
\text { Exploratório } \\
\text { Método } \\
\text { Quantitativo }\end{array}$ & $\begin{array}{c}\text { Caracterizar o nível funcional e de } \\
\text { dependência na alta da UCl e verificar } \\
\text { momento do início das intervenções de } \\
\text { enfermagem de reabilitação. } \mathrm{N}=30\end{array}$ & $\begin{array}{l}\text { Dos } 10 \text { itens totais, } 6 \\
\text { têm resposta positiva }\end{array}$ \\
\hline E4 & $\begin{array}{l}\text { Mudge, et } \\
\text { al. } 2020\end{array}$ & Austrália & $\begin{array}{l}\text { Estudo } \\
\text { Descritivo } \\
\text { Transversal }\end{array}$ & $\begin{array}{l}\text { Procurar entender as perspetivas da equipa } \\
\text { multidisciplinar sobre barreiras e facilidades } \\
\text { da mobilidade em locais de reabilitação. } \mathrm{N}= \\
83\end{array}$ & $\begin{array}{l}\text { Dos } 8 \text { itens totais, } 6 \text { têm } \\
\text { resposta positiva }\end{array}$ \\
\hline E5 & $\begin{array}{l}\text { Tran, et al. } \\
2020\end{array}$ & EUA & $\begin{array}{l}\text { Estudo de } \\
\text { Coorte } \\
\text { Retrospetivo }\end{array}$ & $\begin{array}{l}\text { Comparação da relação entre o status de } \\
\text { ambulatório e o status de disposição (casa } \\
\text { vs instalação de reabilitação aguda, casa de } \\
\text { repouso, readmissão numa UCl ou morte) } \\
\text { em termos de cuidados médicos e de } \\
\text { reabilitação precoce em doentes com } \\
\text { internamento prolongado na UCl. } \mathrm{N}=285\end{array}$ & $\begin{array}{l}\text { Dos } 11 \text { itens totais, } 6 \\
\text { têm resposta positiva }\end{array}$ \\
\hline
\end{tabular}

A tipologia dos artigos mais representativa foi o estudo de revisão sistemática da literatura. Portugal é representado por dois artigos desta amostra. As amostras dos estudos têm uma grande amplitude de participantes variando de 25 a 541 .

Na tabela 3 são apresentados os principais resultados e conclusões dos estudos identificados.

Tabela 3 - Resultados dos estudos identificados

\begin{tabular}{|c|c|c|}
\hline $\begin{array}{l}\text { Código do } \\
\text { estudo }\end{array}$ & Resultados & Conclusões \\
\hline $\mathrm{E} 1$ & $\begin{array}{l}\text { O conceito de mobilização precoce ainda não se } \\
\text { encontra bem definido. No entanto, sabe-se que esta } \\
\text { ajuda a prevenir o declínio cognitivo e funcional e } \\
\text { estimula o funcionamento muscular, melhora o humor e a } \\
\text { autoestima da amostra e, evita o aparecimento de } \\
\text { complicações devido à inatividade prolongada. }\end{array}$ & $\begin{array}{l}\text { A mobilização precoce é essencial para restaurar a } \\
\text { saúde após a hospitalização. O resultado do estudo é } \\
\text { condicionado por variáveis relacionadas com a amostra, } \\
\text { a equipa, modelo organizacional e o contexto de } \\
\text { cuidado. São necessárias mais pesquisas para planear } \\
\text { e avaliar a eficácia de programas de reabilitação e riscos } \\
\text { para os idosos em risco de síndrome de desuso. }\end{array}$ \\
\hline E2 & $\begin{array}{l}\text { A mobilidade precoce pode facilitar a reabilitação } \\
\text { funcional dos doentes, promovendo ganhos de força } \\
\text { muscular e maior participação nas atividades de vida } \\
\text { diárias. }\end{array}$ & $\begin{array}{l}\text { São necessários estudos que usem as mesmas } \\
\text { ferramentas de avaliação e que demonstrem o interesse } \\
\text { desta intervenção para a enfermagem. }\end{array}$ \\
\hline E3 & $\begin{array}{l}\text { O valor médio da MRC (Medical Research Council) foi de } \\
27 \text { na } 1^{\text {a }} \text { avaliação e de } 38 \text { na alta. O valor médio de MIF } \\
\text { (Medição de Independência Funcional) no autocuidado, } \\
\text { mobilidade/transferência foi de } 2 . \text { A } 1^{\text {a }} \text { intervenção de } \\
\text { enfermagem de reabilitação ocorreu em média no } 10^{\circ} \\
\text { dia e o } 1^{\circ} \text { levante após } 13 \text { dias de internamento. }\end{array}$ & $\begin{array}{l}\text { No momento da alta verificaram-se elevados níveis de } \\
\text { dependência funcional nos autocuidados básicos e } \\
\text { mobilidade/transferência, sendo necessário referenciar } \\
\text { para continuidade de cuidados de enfermagem de } \\
\text { reabilitação. A mobilização de doentes críticos deve } \\
\text { ocorrer o mais precocemente possível. }\end{array}$ \\
\hline $\mathrm{E} 4$ & $\begin{array}{l}\text { As principais barreiras identificadas foram a carga de } \\
\text { trabalho dos enfermeiros, a pouca clareza quanto à } \\
\text { responsabilidade da mobilização, os riscos de lesões da } \\
\text { equipa, a motivação do paciente e a participação da } \\
\text { família. Fatores facilitadores: expectativas de resultados } \\
\text { positivos e boa comunicação entre a equipa. }\end{array}$ & $\begin{array}{l}\text { Sugerem potenciais estratégias para melhorar a } \\
\text { mobilidade e reconhecem o dano da imobilidade } \\
\text { incentivando a mobilidade precoce. }\end{array}$ \\
\hline E5 & $\begin{array}{l}\text { Dos } 285 \text { participantes, } 68 \% \text { foram transferidos de UCl. A } \\
\text { média de permanência nas UCI e em hospitais de } \\
\text { cuidados agudos de longa duração foi de } 25.5 \text { e de } 34 \\
\text { dias, respetivamente. } 38 \text { dos participantes foram para }\end{array}$ & $\begin{array}{l}\text { A capacidade de deambulação está associada a uma } \\
\text { maior probabilidade de terem alta para o domicílio } \\
\text { depois de sobreviverem a um internamento prolongado } \\
\text { na UCl e admitidos em unidades de cuidados de longa }\end{array}$ \\
\hline
\end{tabular}


No estudo (Tran, et al., 2020) dos 285 participantes, 68\% foram transferidos de UCI. A média de permanência nas UCI e em hospitais de cuidados agudos de longa duração foi de 25.5 e de 34 dias, respetivamente. Do total de participantes, 38 foram para casa, 25 foram para instalações de reabilitação de agudos, 70 tinham enfermagem domiciliária, 139 foram readmitidos na UCl e 13 morreram. Da mesma amostra, 74 deambularam durante a fisioterapia dos quais 24 foram para casa, enquanto 211 nunca deambularam e apenas 14 regressaram ao domicílio.

O estudo de Azevedo et al. (2019), usou a escala MRC, cujo valor médio da MRC foi de 27 na $1^{\text {a }}$ avaliação e de 38 na alta. Assim como o valor médio de MIF no autocuidado, mobilidade/transferência foi de 2 . No momento da alta verificaram-se elevados níveis de dependência funcional nos autocuidados básicos e mobilidade/transferência, sendo necessário referenciar para continuidade de cuidados de enfermagem de reabilitacão. A $1^{\mathrm{a}}$ intervencão de enfermagem de reabilitação ocorreu em média no $10^{\circ}$ dia e $01^{\circ}$ levante após 13 dias de internamento (Azevedo, et al., 2019). Segundo Azevedo \& Gomes (2015), são necessários estudos que usem as mesmas ferramentas de avaliação e que demonstrem o interesse desta intervenção para a enfermagem.

No estudo de Mudge et al., (2020), os autores referem que as principais barreiras identificadas foram: a carga de trabalho dos enfermeiros, a pouca clareza quanto à responsabilidade da mobilização, os riscos de lesões da equipa, a motivação do paciente e a participação da família. Como fatores facilitadores podemos extrair as expectativas de resultados positivos e a boa comunicação entre os elementos da equipa. (Mudge, Alison et al., 2020). Por outro lado, Tempera, Alfio et al. (2013) referem que os resultados dos estudos são condicionados por variáveis relacionadas com a amostra, a equipa, modelo organizacional e o contexto de cuidados.

\section{DISCUSSÃO}

A transição para a dependência no autocuidado, segundo Meleis (2010), é um fator modificável através da melhoria do potencial de aprendiżagem da pessoa, no qual os enfermeiros de reabilitação podem contribuir de forma significativa quer na promoção do autocuidado, quer na qualidade dos processos de transição vividos pelas pessoas, através de medidas contínuas em que toda a equipa de saúde deve estar envolvida e, onde o enfermeiro de reabilitação pode constituir uma mais-valia. Num internamento prolongado em Cuidados Intensivos compete ao enfermeiro de reabilitação avaliar a funcionalidade, conceber e implementar programas de treino motor, cardíaco e respiratório e diagnosticar alterações que determina as limitações da atividade e incapacidade (amplitude articular, força muscular, equilíbrio, necessidades funcionais e exame físico) para capacitá-lo para o autocuidado (higiene, vestir e despir, uso sanitário, arranjar-se, transferência e deambulação) (DR, 2019; OE, 2018).

0 artigo de Azevedo, et al. (2019), demonstra que a intervenção do enfermeiro de reabilitação (décimo dia) permitiu que o primeiro levante fosse efetuado apos três dias do início da sua intervenção. 0 que nos leva a concluir que quanto mais precoce for a intervenção do enfermeiro de reabilitação mais rapidamente obtemos ganhos em saúde. Também Beliz, Bule e Sousa (2020) verificaram que houve ganhos em alguns movimentos articulares e que a realização de exercícios passivos de mobilização articular não interferiu com a estabilidade de parâmetros fisiológicos. Assim como, o ganho em saúde e a minimização das complicações associadas à imobilidade, devido à implementação de um plano estruturado e regular das mobilizações pelos enfermeiros de reabilitação.

Tran, Maheshwari, Nagaria, Patel, Verceles (2020) referem que a mobilização precoce está associada a melhores resultados funcionais e a baixas readmissões de doentes hospitalizados. Portanto, quando o doente é internado em cuidados intensivos deverá ser iniciado, desde logo, a preparação para a alta, tendo em conta o princípio da mobilização precoce e todo o seu processo de reabilitação. Ao contrảio, quando não se inicia desde logo a mobilização em doentes acamados, atingimos rapidamente a síndrome do desuso e Azevedo et al., (2019) referem que a perda de massa muscular ocorre durante a primeira semana e está diretamente relacionada com a falência multiorgânica. Azevedo et al., (2019) referem ainda que, a recuperação de doentes que estiveram em cuidados intensivos pode levar semanas, meses e persistir até 2 anos após a alta. Assim, iniciar a reabilitação direcionada à mobilidade no início do plano de cuidados destes doentes, não é apenas importante para prevenir a perda muscular e subsequente imobilidade, mas também é um aspeto crítico necessário para melhorar os resultados e a qualidade geral de vida. Segundo Gil, Sousa e Martins (2020) são evidenciados ganhos no equilíbrio postural e na fragilidade após implementação de 9 sessões de exercícios terapêuticos e treino de equilíbrio. Essa a implementação de cuidados de enfermagem de reabilitação revela-se eficaz na recuperação do equilíbrio corporal na pessoa com síndrome do desuso, assim como na melhoria da fragilidade, otimizando a sua autonomia.

Pelo que, há necessidade de elaborar e implementar programas de treino de atividades de vida diárias visando a adaptacão às limitações da mobilidade e à maximização da autonomia e da qualidade de vida e promover a mobilidade, a acessibilidade deambular e a participação social. Tendo-se verificado que é um fator determinante no regresso ao domicílio (Tran, et al. 2020) e que é necessário a elaboração de mais programas de reabilitação para reduzir os riscos (Tempera, et al. 2013).

Desses estudos analisados acerca da mobilização precoce, o que obtivemos permite-nos reportar que esta, ajuda a prevenir o declínio cognitivo e funcional e estimula o funcionamento muscular, assim como melhora o humor e a autoestima e, evita o aparecimento de complicações devido à inatividade prolongada. Facilitar a reabilitação funcional dos doentes, promove ganhos de força muscular e uma maior participação destes nas suas atividades de vida diárias.

Para o enfermeiro especialista em reabilitação intervir na mobilidade da pessoa que cuida, inicia pela avaliação da funcionalidade, das limitações e incapacidades, bem como uma observação dos segmentos que interferem no movimento da pessoa, mas também um conhecimento dos dados clínicos e historia de vida que possa influenciar os movimentos ou a massa muscular e estruturas dessa pessoa, só depois faz o diagnostico e inicia o seu plano de trabalho onde programa as intervenções necessárias para melhorar a mobilidade da pessoa e prevenir as consequências da imobilidade (OE, 2018). A ações do enfermeiro será pautado de programação diária com uma progressiva frequência e intensidade dos movimentos setoriais e numa segunda fase movimentos mais complexos que progressivamente levam ao autocuidado como é o caso de andar. Facilmente aceitamos que não é suficiente a intervenção 
apenas do enfermeiro de reabilitação, a mobilidade do doente é uma área que se executa durante as vinte e quatro horas logo resulta em muito de um trabalho de uma equipe mais alargada, que lucrará com a gestão do cuidado do enfermeiro de reabilitação.

Após determinar as limitações e incapacidades dos doentes, o enfermeiro de reabilitação, de acordo com as suas competências, concebe um plano de intervenção com vista ao autocontrolo e autocuidado nos processos de transição saúde/doença e ou incapacidade, implicando uma reeducação funcional e/ou readaptação de modo a minimizar os efeitos da incapacidade, com vista à sua reinserção e exercício de cidadania (Ordem dos enfermeiros, 2019). Ainda segundo a Ordem dos enfermeiros (2019), a implementação das intervenções, tendo em conta as competências do enfermeiro de reabilitação, tem como objetivo otimizar e/ou reeducar as funções aos níveis motor, sensorial, cognitivo, cardíaco, respiratório, eliminação e sexualidade. Deve também, incidir a atividade na função/estrutura do corpo através da reeducação e readaptação funcional, de forma a evitar e corrigir alterações posturais e defeitos ventilatórios, manter a permeabilidade das vias aéreas, reeducar a pessoa ao esforço e consciencializar sobre a respiração.

Tal como o artigo de Azevedo \& Gomes, (2015) nos demonstra, o plano de intervenção deve ser aplicado o mais precocemente possível, de modo a evitar o aparecimento de complicaç̃es (perda de massa muscular, perda de capacidade de equilíbrio e de marcha, dificuldade respiratória, edemas, alterações gastrointestinais e alterações genito-urinárias). Cerol, Martins, Sousa, Oliveira \& Silveira (2019) realçam os resultados do estudo em análise, já que constataram que os principais benefícios da mobilização precoce são a melhoria da capacidade funcional, a diminuição das complicações, a redução do número de dias sob ventilação mecânica invasiva e consequentemente a diminuição de permanência na unidade de cuidados intensivos e de internamento hospitalar. Esta intervenção diminui a morbilidade e mortalidade hospitalar.

Durante este processo, existem barreiras que tanto podem ser facilitadoras como inibidoras de uma mobilização precoce (Mudge, Alison et al., 2020), cabendo ao enfermeiro de reabilitação a sua desmitificação. O que corrobora com o estudo de Cerol, Martins, Sousa, Oliveira e Silveira (2019) que identificaram como barreiras a sedação e a entubação endotraqueal e realçaram a utilização de protocolos para minimizar essas barreiras, adequando a gestão do nível de sedação, de forma à pessoa ter capacidade de se mobilizar precocemente e cooperar com as intervenções a realizar.

\section{CONCLUSÃO}

Da análise efetuada podemos concluir que a mobilização precoce é essencial para restaurar a saúde após a hospitalização. Assim, esta mobilização em doentes críticos deve ocorrer o mais precocemente possível. E, a existência de protocolos de reabilitação com enfase na mob̉ilização precoce pode facilitar a otimização das intervenções permitindo obter ganhos mais rapidamente possível. A enfermagem tem aqui um papel importante devido aos seus conhecimentos e habilidades, uma vez que estes ganhos são obtidos através de atividades especificas da reabilitação.

A capacidade de deambulação depende da mobilidade da pessoa e está associada a uma maior probabilidade de terem alta para o domićlio depois de sobreviverem a um internamento prolongado na UCl e admitidos em unidades de cuidados de longa duração. O treino da mobilidade é importante para o regresso a casa. Do ponto de vista da enfermagem de reabilitação não basta apenas que se evidenciem ganhos físicos, mas que também contribuam para melhor desempenho das atividades de vida diária, no sentido de readquirir a máxima independência possível, ou seja torna-se necessário a sua readaptação e reeducação funcional, de modo, a que haja também uma total integração na família, na sociedade e no seu papel de cidadania.

São necessárias mais pesquisas para planear e avaliar a eficácia de programas de reabilitação para os doentes internados em unidades de cuidados intensivos em risco da síndrome de desuso. Assim como, usar sempre as mesmas ferramentas de avaliação de forma a demonstrarem o interesse desta intervenção para a enfermagem.

Sugerimos potenciais estratégias para melhorar a mobilidade e que se reconheça as consequências da imobilidade, usando um programa que permita uma avaliação por parte da equipa multidisciplinar, das intervenções necessárias para promover a mobilidade precoce dos doentes internados e, que os próprios doentes se sintam motivados a participar.

\section{DIVULGAÇÕES ÉTICAS}

\section{Contribuição dos autores:}

Conceptualização: CS, CS, CE, RS, MM, CF;

Metodologia: CS, CS, CE, RS;

Validação: CS,CS, CE, RS;

Análise formal: CS, CS, CE, RS;

Investigação: CS, CS, CE, RS;

Tratamento de dados: CS, CS, CE, RS;

Preparação do rascunho original: CS, CS, CE, RS;

Redação e edição: CS, CS, CE, RS;

Revisão: CS, CS, CE, RS;

Todos os autores leram e concordaram com a versão publicada do manuscrito.

Financiamento: 
Este trabalho não recebeu nenhuma contribuição financeira ou bolsa.

Comissão de Ética:

Não se aplica.

Declaração de consentimento informado:

Não se aplica.

Conflitos de interesse:

Os autores não declaram nenhum conflito de interesses.

Proveniência e revisão por pares:

Não comissionado; revisto externamente por pares.

\section{Referências}

1. Azevedo, P., Gomes, B. (2015) - Efeitos da mobilização precoce na reabilitação funcional em doentes críticos: uma revisão sistemática. Enfermagem Referência, IV (5), 129-138. Retirado de http://dx.doi.org/10.12707/RIV14035

2. Azevedo, P., Gomes, B., Pereira, J., Carvalho, F., Ferreira, S., Pires, A., Macedo, J. (2019) - Dependência funcional na alta dos cuidados intensivos: relevância para a enfermagem de reabilitação. Enfermagem Referência, IV (20), 129-138. Retirado de http://dx.doi.org/10.12707/RIV18084

3. Beliz, A., Bule, M., Sousa, L. (2020) - Manter a mobilidade articular no doente crítico: estudo de caso. Revista Portuguesa de Enfermagem de Reabilitação, 3 (S1), 63-69

4. Cerol, P., Martins, J., Sousa, L., Oliveira, I., Silveira, T. (2019) - Mobilização precoce em pessoas submetidas a ventilação mecânica invasive: revisão integrativa da Literatura. Revista Portuguesa de Enfermagem de Reabilitação, 2(1), 49-58

5. Diário da república. (2019). Regulamento das competências específicas do enfermeiro especialista em Enfermagem de Reabilitação, regulamento 329. Lisboa: DGS. Disponível em https://dre.pt/application/conteudo/122216893

6. Fortin, M., Côté, J, Filion, F (2009) - Fundamentos e etapas do processo de investigação. Loures, Portugal: Lusodidata.

7. Gil, A., Sousa, F., Martins, M. (2020) - Implementação de programa de enfermagem de reabilitação em idoso com fragilidade/sindrome de desuso - estudo de caso. Revista Portuguesa de Enfermagem de Reabilitação, 3 (2), $27-35$

8. Gomes, J., Martins, M., Gonçalves, M., Fernandes, C. (2012) -Enfermagem de reabiltação: pecurso para a avaliação da qualidade em unidades de internamento. Revista de enfermagem de referência. III (8), 29-38. Retirado de http: //www.scielo.mec.pt/pdf/res/vserlllno/serllnn8a03.pdf

9. Karic, T., Røe, C., Nordenmark, T. H., Becker, F., \& Sorteberg, A. (2016). Impact of early mobilization and rehabilitation on global functional outcome one year after aneurysmal subarachnoid hemorrhage. Journal of Rehabilitation Medicine, 48(8), 676-682. https://doi.org/10.2340/16501977-2121

10. Marques, P.A.O., Sousa, P.A.F., \& Silva, A.A.P. (2013). Confusão Aguda no idoso: Dados para a decisão do enfermeiro. Revista de Enfermagem Referência, 3(9), 37-43. https://doi.org/10.12707/RIII1292

11. Meleis, A. (2010). Transitation Theory: Middle range and situation specific theories in nursing research and practice. Springer: Publishing Company. New York.

12. Mudge A., Bew, P., Smith S., McRae P. (2020) - Staff knowledge, attitudes and behaviours related to mobilisation in a rehabilitation setting: Short report of a multidisciplinary survey. Australasian Journal on Ageing, 00, 1 - 5. Retirado de https: //doi.org/10.1111/ajagg.12793

13. Ordem dos Enfermeiros (2013) - Guia Orientador de Boas Práticas: Cuidados à pessoa com alterações da mobilidade: posicionamentos, transferências e treino de deambulação. Lisboa: Ordem dos Enfermeiros. Disponível em: http://www.ordemenfermeiros.pt/publicacoes/documents/gobp_mobilidade vf site.pdf

14. Ordem dos Enfermeiros (2018) - Padrões de qualidade dos cuidados especializados em enfermagem de reabilitação. Lisboa, Colégio da especialidade de enfermagem de reabilitação.

15. Ordem dos Enfermeiros. (2011). Regulamento dos Padrões de Qualidade dos Cuidados Especializados em Enfermagem de Reabilitação. Lisboa: Ordem dos Enfermeiros .

16. Tempera A., Re Luca G, Lusignani M. (2013) - La mobilizzazione precoce per la prevenzione della sindrome da allettamento prolungato nell'utente anziano ospedalizzato: una revisione della letteratura. L'infermiere, 57 (4), 63-73.

17. Tran, D., Maheshwari, P., Nagaria, Z., Patel, H., Verceles A. (2020) - Ambulatory Status Is Associated With Successful Discharge Home in Survivors of Critical Illness. Respiratory Care, 65 (8), $1168-117$

18. Wang, J., Xiao, Q., Zhang, C., Jia, Y., \& Shi, C. (2020). Intensive care unit nurses' knowledge, attitudes, and perceived barriers regarding early mobilization of patients. Nursing in Critical Care, 25(6), 339-345. https://doi.org/10.1111/nicc.12507 


\section{(ف) $19 \Theta$}

Este trabalho encontra-se publicado com a Licença Internacional Creative Commons Atribuição-NãoComercial-SemDerivações 4.0.

Direitos de Autor (c) 2021 Revista Portuguesa de Enfermagem de Reabilitação 